\title{
Longer Weekly Sleep Duration Predicts Greater 3-Month BMI Reduction among Obese Adolescents Attending a Clinical Multidisciplinary Weight Management Program
}

\author{
Bethany J. Sallinen $^{a}$ Fauziya Hassan $^{b}$ Amy Olszewskic ${ }^{c}$ Angela Maupin $^{d}$ \\ Timothy F. Hoban $^{\mathrm{e}}$ Ronald D. Chervin ${ }^{f}$ Susan J. Woolford ${ }^{g}$ \\ aPediatric Comprehensive Weight Management Center, Department of Pediatrics and \\ Communicable Diseases, Division of Child Behavioral Health, bediatric Pulmonary and Sleep, \\ Sleep Disorders Center, University of Michigan, Ann Arbor, MI, ' Eastern Michigan University, \\ Ypsilanti, MI, ${ }^{\mathrm{d}}$ Michigan State University, East Lansing, MI, ${ }^{\mathrm{e}}$ Department of Pediatrics and \\ Communicable Diseases, Department of Neurology, Sleep Disorders Center, ${ }^{\mathrm{f}}$ Department of \\ Neurology, Sleep Disorders Center, 9 Pediatric Comprehensive Weight Management Center, \\ Department of Pediatrics and Communicable Diseases, Child Health Evaluation and Research \\ (CHEAR) Unit, University of Michigan, Ann Arbor, MI, USA
}

Key Words

Obesity $\cdot$ Adolescent $\cdot$ Sleep $\cdot$ Weight loss $\cdot$ Treatment

\begin{abstract}
Aims: To determine whether baseline levels of self-reported sleep and sleep problems among obese adolescents referred to an outpatient multidisciplinary family-based weight management program predict reduction in BMI 3 months later. Methods: A retrospective medical chart review was conducted for 83 obese adolescents. The following baseline variables were extracted: self-reported sleep duration (weekdays and weekends), and presence of snoring, daytime fatigue, suspected sleep apnea, and physician-diagnosed sleep apnea. Anthropometric data at baseline and 3 months were also collected. Results: On average, adolescents reported significantly less sleeping on weeknights $(7.7 \pm 1.3 \mathrm{~h}$ ) compared to weekend nights $(10.0 \pm 1.8 \mathrm{~h}), \mathrm{t}(82)=10.5, \mathrm{p}=0.0001$. Reduction in BMI after 3 months of treatment was predicted by more weekly sleep at baseline $\left(R^{2}=0.113, F(1,80)=10.2, p=0.002\right)$. Adolescents who reduced their BMI by $\geq 1 \mathrm{~kg} / \mathrm{m}^{2}$ reported greater weekly sleep at baseline compared to adolescents who experienced $<1 \mathrm{~kg} / \mathrm{m}^{2}$ reduction $(60.7 \pm 7.5 \mathrm{~h}$ vs. $56.4 \pm 8.6 \mathrm{~h} ; \mathrm{F}(1,80)=5.7$, $p=0.02$ ). Conclusion: Findings from this study, though correlational, raise the possibility that increased duration of sleep may be associated with weight loss among obese adolescents enrolled in a weight management program. Evidence-based behavioral techniques to improve sleep hygiene and increase sleep duration should be explored in pediatric weight management settings.


Sallinen et al:: Longer Weekly Sleep Duration Predicts Greater 3-Month BMI Reduction among Obese Adolescents Attending a Clinical Multidisciplinary Weight Management ...

\section{Introduction}

According to data collected from the National Health and Nutrition Examination Survey during 2003-2006, prevalence estimates of high BMI suggest that $15.6 \%$ of children aged $2-19$ years are overweight and $16.3 \%$ are obese [1]. As such, there is a pronounced need for effective pediatric weight management programs. To date, effective programs generally involve a family-based behavioral approach that fosters healthy dietary changes, increases physical activity, and decreases sedentary behavior [for review see 2]. Although reduction in weight status has been demonstrated, the effects of behavioral weight management programs are not particularly robust. Therefore, any opportunity to increase effectiveness of existing interventions merits careful exploration.

One potential intervention target may be sleep. A meta-analysis by Hart and Jelalian [3] that included results from cross-sectional as well as longitudinal studies demonstrated that short sleep duration is associated with an increased likelihood of being overweight or obese in childhood. Although an association between obesity and shortened sleep duration appears to exist, the specific factors that influence this relationship remain unknown. In studies on adults it has been demonstrated that short sleep duration affects levels of the hormones ghrelin and leptin, which are involved in the regulation of hunger and appetite [4-5]. In children, short sleep duration, independent of weight status, has been shown to be associated with insulin resistance, higher fasting insulin, and peak insulin [6]. These results highlight the need to better understand neuroendocrine factors that may be driving the relationship between short sleep duration and obesity risk in youth.

Not only is short sleep duration associated with obesity in children, but there is also an increased prevalence of breathing dysfunctions associated with sleep disorders. For example, prevalence estimates suggest that up to $26 \%$ of obese children in weight management programs have obstructive sleep apnea (OSA), as diagnosed by polysomnography $[7,8]$. In a study examining sleep differences in obese children presenting to weight management treatment compared to healthy controls, it was found that the obese group fell asleep later and experienced shorter, more disrupted sleep as measured by actigraphy [9]. In addition, parent report of sleep concerns, including daytime sleepiness, parasomnias, bedtime resistance and delayed sleep onset, were greater in the obese group. While untreated OSA may influence sleep duration, it is unknown to what extent OSA or other sleep-related problems, such as daytime sleepiness, may account for the association between short sleep duration and obesity in children.

While evidence is mounting to suggest a relationship between short sleep duration and obesity, the effect of sleep duration on weight loss in obese youth is unexplored. The primary aim of the study was to conduct a retrospective medical chart review of obese adolescents who participated in a clinical, family-based, multidisciplinary, weight management program to determine whether self-reported sleep and sleep problems at entry to the program, presumed to persist at least somewhat during treatment, predicted reduction in BMI 3 months later. Given the link between short sleep duration and obesity, we hypothesized that obese youth with shorter sleep duration would experience less weight loss compared to obese youth with greater sleep duration, after controlling for OSA.

\section{Participants and Methods}

\section{Participants}

Participants were 83 obese adolescents referred for weight management between January 2008 and January 2011. Inclusion criteria for the clinical program were: i) age $12-18$ years, and ii) BMI (kg/m²) equal to or greater than the 95th percentile for age and gender using Center for Disease Control (CDC) growth 
Table 1. Standard sleep questions

1) Over the past week, approximately what time did you typically get in bed on a school night?

Approximately what time did you fall asleep? Approximately what time did you wake up?

2) Over the past week, what time did you typically get in bed on weekend nights (i.e., Friday and

Saturday)? What time did you typically fall asleep? What time did you wake up?

3) Do you snore?

4) Do you feel sleepy or tired during the day (i.e., daytime fatigue)?

5) Do you have pauses in your breathing while sleeping?

6) Do you have any concerns about your sleep?

7) Have you ever been diagnosed with obstructive sleep apnea?

8) Has a physician ever suspected obstructive sleep apnea?

charts [10]. Three participants were dropped from the analyses due to a diagnosis of autism and receipt of a different treatment protocol tailored to their mental health needs.

\section{Procedure}

A retrospective medical chart review was performed to examine self-reported sleep duration and sleeprelated problems. At the initial consultation the pediatric psychologist asked each patient, in the presence of the parent or legal guardian, a series of standard questions related to sleep (please see table 1 for specific questions). If the parent or guardian expressed disagreement with the adolescent's response, he or she participated in a discussion facilitated by the psychologist until agreement was met. The following variables were extracted from the pediatric psychologist's documentation in the medical record: number of hours of weeknight sleep, number of hours of weekend night sleep, and presence or absence of snoring, daytime fatigue, suspected sleep apnea (i.e., endorsed 2 of 3 symptoms: snoring, daytime fatigue, and apneic episodes), and physician diagnosed OSA. Anthropometric data at baseline and 3 months were collected for those patients who joined and participated in the weekly 2-hour program for at least 3 months. Body weight (0.1 $\mathrm{kg}$ ) was measured via a calibrated electronic scale. Height was measured to the nearest $0.1 \mathrm{~cm}$ using a wallmounted stadiometer. BMI $\left(\mathrm{kg} / \mathrm{m}^{2}\right)$ was then calculated from weight and height measurements.

\section{Program Description}

The Michigan Pediatric Outpatient Weight Evaluation and Reduction (MPOWER) program is a 24-week, clinical, family-based weight management program delivered by a multidisciplinary team of providers. The program features weekly 1-hour exercise group sessions led by an exercise physiologist and 1-hour group or individual sessions. Group topics address nutrition education and behavioral skills to assist with weight management efforts. Parents and adolescents attend nutrition sessions together, led by a dietitian, whereas behavioral sessions are conducted in separate but simultaneous adolescent and parent groups by a pediatric psychologist (i.e., parent group) and social worker (i.e., adolescent group). Individual sessions focus on assessment of knowledge acquisition and application of skills/knowledge, adherence to goals and program recommendations, motivation, and assessment of factors contributing to weight loss or weight gain. Please refer to Woolford et al. [11] for further program details.

\section{Statistical Analyses}

Descriptive statistics were calculated for all demographic and sleep variables. Sleep duration was computed as the difference between estimated sleep onset and rise time. Weekly sleep was estimated by the following equation:

Weekly sleep $=($ hours weekday night sleep $\times 5)+($ hours weekend night sleep $\times 2)$.

Correlational analyses were conducted to explore relationships among all sleep, demographic, and anthropometric variables. Linear regression was used to examine sleep variable predictors of BMI reduction at 3 months. Analysis of variance (ANOVA) was used to calculate differences in baseline weekly sleep duration for adolescents who reduced their BMI after 3 months of treatment by at least one unit $\left(1.0 \mathrm{~kg} / \mathrm{m}^{2}\right)$ versus those who did not. A logistic regression model was then used to test for moderation effects. All analyses were conducted using SPSS 17.0 (SPSS Inc.; Chicago, IL, USA). This retrospective chart review was approved by the Institutional Review Board of the University of Michigan Medical School. 
Table 2. Percentage of obese adolescents endorsing sleep problems

\begin{tabular}{l|l}
\hline Obes Facts 2013;6:239-246 \\
\hline DOI: 10.1159/000351819 & $\begin{array}{l}\text { @ } 2013 \text { S. Karger GmbH, Freiburg } \\
\text { www.karger.com/ofa }\end{array}$ \\
\hline
\end{tabular}

Sallinen et al.: Longer Weekly Sleep Duration Predicts Greater 3-Month BMI Reduction among Obese Adolescents Attending a Clinical Multidisciplinary Weight Management ...

\begin{tabular}{ll}
\hline & $\%$ \\
\hline Snoring & 43.9 \\
Daytime fatigue & 40.7 \\
Suspected obstructive sleep apnea & 19.7 \\
Obstructive sleep apnea diagnosis & 7.4 \\
\hline
\end{tabular}

Fig. 1. Association between hours of self-reported weekly sleep at baseline and change in BMI (kg/ m2) after 3 months of weight management treatment.

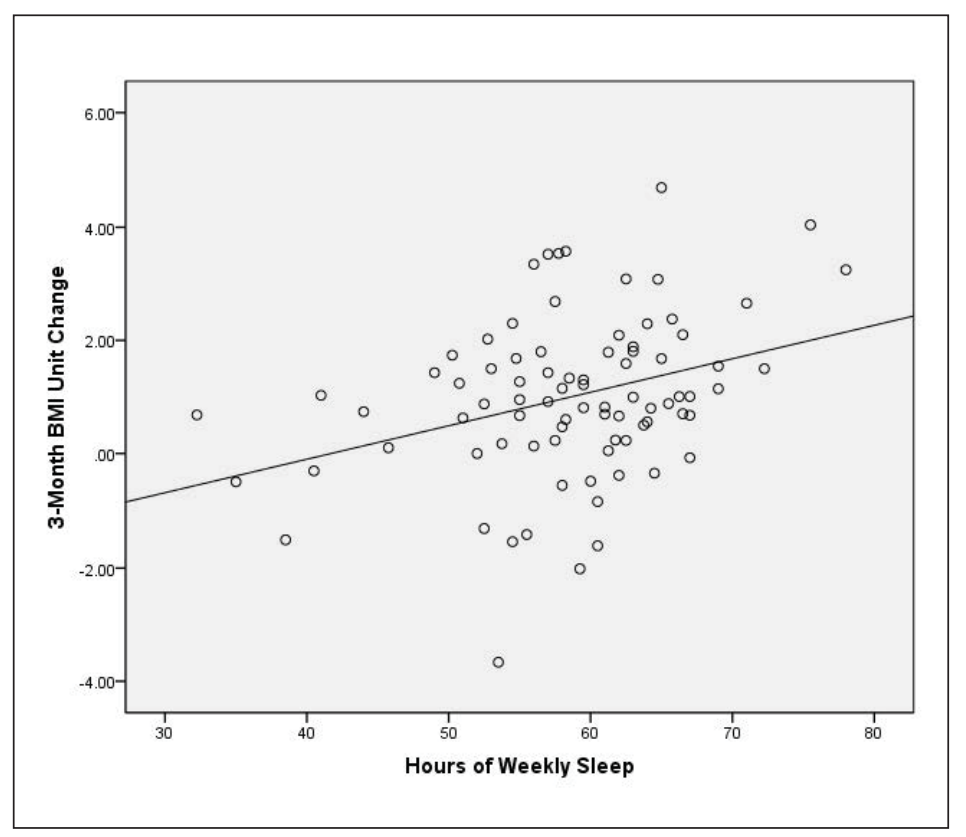

\section{Results}

The mean age of adolescents was 14.5 years (range 12-18-years), and the mean baseline BMI was $43.4 \mathrm{~kg} / \mathrm{m}^{2}\left(27.4-78.3 \mathrm{~kg} / \mathrm{m}^{2}\right)$. The majority were female $(72.3 \%)$, white (59\%), and carriers of private insurance (65.1\%). On average, adolescents reported sleeping significantly less on weeknights $(7.7 \pm 1.3 \mathrm{~h})$ compared to weekend nights $(10.0 \pm 1.8 \mathrm{~h}), \mathrm{t}(82)=10.5, \mathrm{p}=$ 0.0001 . The percentage of adolescents who endorsed one or more sleep-related problems was $57 \%$ (table 2).

Reduction in BMI after 3 months of weight management program participation was predicted by more weekly sleep at baseline $\left(\mathrm{R}^{2}=0.113, \mathrm{~F}(1,80)=10.2, \mathrm{p}=0.002\right.$; fig. 1$)$. Weekly sleep was correlated inversely with daytime fatigue $(r=-0.34, p=0.002)$ and age $(\mathrm{r}=-0.30, \mathrm{p}=0.006)$. Thus, daytime fatigue and age were taken into account in the regression model shown in table 3 . Weekly sleep retained its association with BMI reduction. No other sleep variables, including physician diagnosed OSA, or demographic variables were significantly associated with weekly sleep or BMI reduction at 3 months, and were thus not entered in the regression model. The final model shows that on average, a 30-min increase per day in baseline sleep (i.e., 3.5-hour increase per week) predicted an increase in BMI reduction by 0.2 $\mathrm{kg} / \mathrm{m}^{2}$.

Due to the possibility that a wide range in initial BMI $\left(27.4-78.3 \mathrm{~kg} / \mathrm{m}^{2}\right)$ may have impacted the association between baseline sleep and subsequent BMI reduction, we looked 
Sallinen et al.: Longer Weekly Sleep Duration Predicts Greater 3-Month BMI Reduction among Obese Adolescents Attending a Clinical Multidisciplinary Weight Management ...

Table 3. Summary of regression analysis for baseline variables predicting BMI reduction after 3 months of weight management treatment $(\mathrm{R} 2=0.113, \mathrm{~F}(1,80)=$ $10.2, \mathrm{p}=0.002$ )

\begin{tabular}{llll}
\hline Variable & $\beta$ & $\mathrm{t}$ & $\mathrm{p}$ \\
\hline Weekly sleep & 0.06 & 3.22 & 0.002 \\
Daytime fatigue & 0.13 & 1.13 & 0.26 \\
Age & 0.14 & 1.24 & 0.22 \\
\hline
\end{tabular}

at percent change in BMI (i.e., 100 - (BMI after 3 months / initial BMI) $\times 100)$ ) and used this as the dependent variable in the regression equation. The association between baseline weekly sleep and 3-month BMI reduction survived $\left(R^{2}=0.122, F(1,80)=11.1, p=0.001\right)$.

At 3 months, the mean BMI reduction for all adolescents in the sample was $1.0 \pm 1.4$ $\mathrm{kg} / \mathrm{m}^{2} .50 \%$ of adolescents experienced a reduction of least $1.0 \mathrm{BMI}$ unit $\left(\mathrm{kg} / \mathrm{m}^{2}\right)$ after 3 months of treatment. Figure 2a shows that adolescents who decreased their BMI by at least 1.0 BMI unit at 3 months reported significantly more weekly sleep at baseline compared to adolescents who did not achieve at least $1.0 \mathrm{BMI}$ unit reduction $(60.7 \pm 7.5 \mathrm{~h}$ vs. $56.4 \pm 8.6 \mathrm{~h}$; $F(1,80)=5.7, p=0.02)$. This effect was then examined using logistic regression to determine if daytime fatigue and/or age modify the relationship. The effect appeared to be modified by self-reported daytime fatigue, but not age (fig. $2 b, c)$. Specifically among adolescents who endorsed daytime fatigue, those who reduced their BMI by at least 1 unit after 3 months of weight management treatment reported significantly more baseline weekly sleep compared to adolescents who did not experience at least a $1.0 \mathrm{BMI}$ reduction $(59.9 \pm 8.3 \mathrm{~h}$ vs. $48.1 \pm 9.0$ $h ; F(1,31)=14.7, p=0.001)$. In contrast, among patients who did not report fatigue, sleep at baseline was not significantly different between patients who did and those who did not experience at least a 1.0 unit reduction in BMI.

\section{Discussion}

This study of 83 obese adolescents suggests that greater levels of self-reported weekly sleep at baseline predict greater reduction in BMI after 3 months of weight management treatment. Although daytime fatigue and age were both associated with weekly sleep, when entered into the regression model, these variables did not contribute significantly to BMI reduction. However, when participants were divided into two groups based on BMI reduction (i.e., $\geq 1$ BMI unit reduction and $<1$ BMI unit reduction), daytime fatigue was found to moderate the relationship. Thus, adolescents who reported daytime fatigue appeared to experience a greater reduction in BMI after 3 months of weight management treatment when they reported higher levels of weekly sleep at baseline.

While evidence has accumulated to support the association between short sleep duration and increased risk of obesity in children, the current findings appear to be the first to suggest that obese youth, who present for weight management treatment with greater self-reported levels of weekly sleep, may achieve a greater reduction in BMI. Reasons for this improved BMI reduction must be speculative at this time, as this study was retrospective in nature and not designed to assess for causality. However, our result is similar to that from a small study conducted with overweight adults who were experimentally sleeprestricted for 14 days in combination with a reduced calorie diet. This combination of $5.5 \mathrm{~h}$ of sleep and a reduced calorie diet led to decreased fat loss, increased fat-free body mass loss, and increased hunger [12]. Previous research in this area suggests the possibility that neuroendocrine factors may play an important role in the association between short sleep duration and obesity [4-6]. 

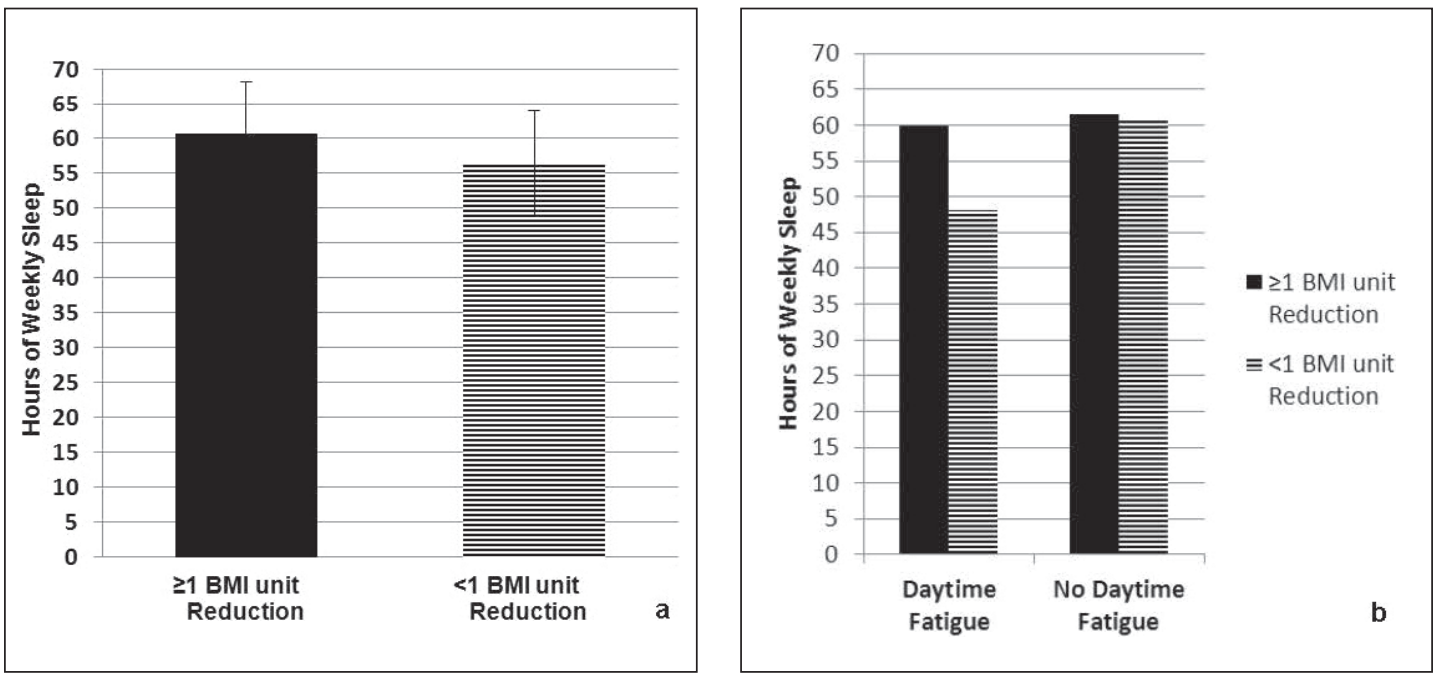

Fig. 2. a Higher levels of self-reported weekly sleep at baseline predicted at least a 1 BMI unit $\left(\mathrm{kg} / \mathrm{m}^{2}\right)$ reduction after 3 months of weight management treatment $(\beta=-0.071, p=0.03)$. $\mathbf{b}$ Endorsement of daytime fatigue ( $\mathrm{n}=33$ of 81 patients) moderates the relationship between self-reported weekly sleep at baseline and achievement of at least a 1 BMI unit $\left(\mathrm{kg} / \mathrm{m}^{2}\right)$ reduction after 3 months of weight management treatment $(\beta=-0.018, p=0.04)$. c Younger age ( $\leq 14$ years, $n=42$ patients) versus older age $(\geq 15$ years, $n=39$ patients) does not moderate the relationship between self-reported weekly sleep at baseline and achievement of at least a 1 BMI unit $\left(\mathrm{kg} / \mathrm{m}^{2}\right)$ reduction after 3 months of weight management treatment $(\beta=-0.015, p=0.06)$.

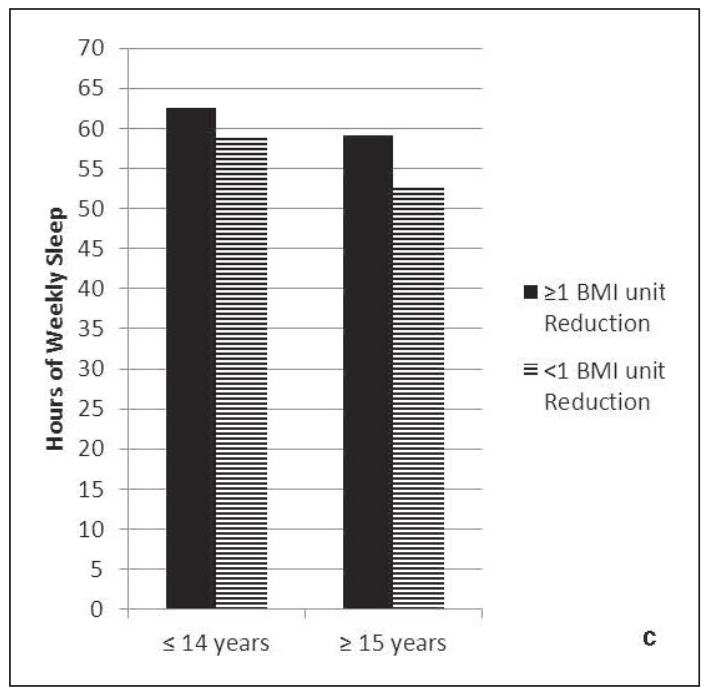

Rates of suspected OSA (19.7\%) and parent-reported OSA diagnosis (7.4\%) appear comparable to results from two studies on prevalence of polysomnography-diagnosed OSA in obese youth presenting to pediatric obesity clinics (i.e., rates ranged from 19 to $26.1 \%$ ) [7, 8]. Symptoms of OSA in our sample were not significantly predictive of BMI reduction. However, given the high prevalence of breathing dysfunctions associated with sleep disorders in obese youth, sleep symptoms should be routinely explored, and youth should be referred for further sleep medicine evaluation if warranted.

One limitation of this study was that it utilized self-reported sleep data based on what was typical for the youth over the past week. Although self-report is subject to recall bias and may not represent what actually occurred, one study has provided evidence to suggest that adolescent self-reported sleep duration over the past 2 weeks is comparable to actigraphy on school nights [13]. While our self-report data may represent 'ideally' what the youth would like to achieve for sleep, parents were present during recall, and the dyads generally appeared to be thoughtful in recalling events that occurred over the past week in order to give the most accurate estimate possible. Furthermore, participants were asked about actual sleep time rather than bedtime. 
A second limitation is that no formal assessment of sleep after 3 months of participation in the program had been done. Therefore, how sleep changed as a result of participation is unknown. However, without direct intervention for sleep habits, differential changes of sleep duration in youth who lost weight versus those who did not are unlikely. Third, this study did not include a no-treatment or wait-list control group for comparison of 3-month BMI change. Fourth, this sample is comprised of youth who were referred to an intensive pediatric weight management program, and these subjects may not represent obese youth from the general population. Lastly, the relationship between short sleep duration and obesity is complex and may be impacted by a number of factors. We did not see any influence of gender or insurance status on sleep and BMI outcomes. However, we did not explore other potential confounders such as family composition, mental health, and social support as there has been no consistent evidence in adolescents to suggest that these factors play a strong role.

Despite these limitations, results from this study suggest that the role of sleep in efforts to reduce weight of obese adolescents merits further research. Assisting adolescents to achieve consistent and healthy sleep durations may improve their weight loss efforts. In fact, a longitudinal study showed that adults with short sleep duration $(\leq 6 \mathrm{~h} /$ day $)$ who were able to increase their sleep duration to 7-8 h/day gained less weight during a 6-year period than did adults who continued to have short sleep durations [14].

Given the potential relationship between longer sleep duration and BMI reduction, sleep habits and hygiene should be routinely assessed in pediatric weight management programs. Behavioral interventions to promote healthy sleep hygiene are well established and can be incorporated into a traditional family-based weight management curriculum. Components of these behavioral sleep interventions typically include stimulus control, self-monitoring, education, and relaxation techniques [15]. Behavioral weight management programs may be perfectly suited to assist youth with healthier sleep habits, as many of the evidence-based behavior change strategies (e.g., self-monitoring, stimulus control, goal setting) that target diet, exercise, and sedentary behavior can also be applied to sleep.

\section{Disclosure Statement}

The authors declared no conflict of interest.

\section{References}

$>1$ Ogden CL, Carroll MD, Flegal KM: High body mass index for age among US children and adolescents, 20032006. JAMA 2008;299:2401-2405.

-2 Luzier JL, Berlin KS, Weeks JW: Behavioral treatment of pediatric obesity: review and future directions. Child Health Care 2010;39:312-334.

3 Hart CN, Jelalian E: Shortened sleep duration is associated with pediatric overweight. Behav Sleep Med 2008; 6:251-167.

4 Taheri S, Lin L, Austin D, Young T, Mignot E: Short sleep duration is associated with reduced leptin, elevated ghrelin, and increased body mass index. PLoS Med. 2004;1:210-217.

5 Leproult R, Van Cauter E: Role of sleep and sleep loss in hormonal release and metabolism. Endocr Dev 2010; 17:11-21.

-6 Flint J, Kothare SV, Zihilkf M, Suarez E, Adams R, Legido A, De Luca F: Association between inadequate sleep and insulin resistance in obese children. J Pediatr 2007;150:364-369.

7 Marcus CL, Curtis S, Koerner CB, Joffe A, Serwint JR, Loughlin GM: Evaluation of pulmonary function and polysomnography in obese children and adolescents. Pediatr Pulmonol 1996;21:176-183.

-8 Wing YK, Hui SH, Pak WM, Ho CK, Cheung A, Li AM, Fok TF: A controlled study of sleep related disordered breathing in obese children. Arch Dis Child 2003;88:1043-1047. 
Sallinen et al.: Longer Weekly Sleep Duration Predicts Greater 3-Month BMI Reduction among Obese Adolescents Attending a Clinical Multidisciplinary Weight Management ...

9 Beebe DW, Lewin D, Zeller M, McCabe M, MacLeod K, Daniels SR, Arnin R: Sleep in overweight adolescents: shorter sleep, poorer sleep quality, sleepiness, and sleep-disordered breathing. J Pediatr Psychol 2007;32: 69-79.

10 Centers for Disease Control and Prevention. Clinical Growth Charts (updated August 4, 2009). www.cdc.gov/ growthcharts/clinical_charts.htm (last accessed may 14, 2013).

$\checkmark 11$ Woolford SJ, Sallinen BJ, Clark SJ, Freed GL: Results from a clinical multidisciplinary weight management program. Clin Pediatr 2011;50:187-191.

12 Nedeltcheva AV, Kilkus JM, Imperial J, Schoeller DA, Penev PD: Insufficient sleep undermines dietary efforts to reduce adiposity. Ann Intern Med 2010;153:435-441.

13 Wolfson AR, Carskadon MA, Acebo C, Seifer R, Gallone G, Labyak SE, Martin JL: Evidence for the validity of a sleep habits survey for adolescents. Sleep 2003;26:213-216.

14 Chaput JP, Despres JP, Bouchard C, Tremblay A: Longer sleep duration associates with lower adiposity gain in adult short sleepers. Int J Obes 2012;36:752-756.

$\checkmark 15$ Mindell JA, Meltzer LJ: Behavioural sleep disorders in children and adolescents. Ann Acad Med Singapore 2008;37:722-728. 